\title{
Relationships among Body Mass Index, Body Image, and Depression in Korean Adults: Korea National Health and Nutrition Examination Survey 2014 and 2016
}

\author{
Gwan Woo Hong ${ }^{1}$, Soo Min Hong ${ }^{2, *}$ \\ ${ }^{1}$ Department of Internal Medicine, Dongguk University Ilsan Hospital, Goyang: ${ }^{2}$ Department of Internal Medicine, Seoul Paik Hospital, Inje University College of \\ Medicine, Seoul, Korea
}

Background: This study extends the findings of our previous examination of the relationship between obesity and depression in Korean adults. Here, we further investigate the relationships among body image perception, weight control method, and depression based on data from the Korea National Health and Nutrition Examination Survey in 2014 and 2016.

Methods: In total, we analyzed 11,782 participants. We classified them into four groups according to body mass index (BMI): underweight (BMl $<18.5 \mathrm{~kg} / \mathrm{m}^{2}$ ), normal-to-overweight (BMl $18.5-24.9 \mathrm{~kg} / \mathrm{m}^{2}$ ), obesity stage I (BMl $\left.25.0-29.9 \mathrm{~kg} / \mathrm{m}^{2}\right)$, and obesity stage II $\left(\mathrm{BMI}>30.0 \mathrm{~kg} / \mathrm{m}^{2}\right)$. Current depression was defined as a score of at least 10 on the nine-item Patient Health Questionnaire. We performed chi-square tests and multivariate logistic regression analyses to examine the relationships among BMI, body image perception, method of weight control, and depression.

Results: Depression was diagnosed in $6.5 \%$ of the study participants. The underweight group had the highest odds ratio (OR) for depression (OR, 1.94; 95\% confidence interval [Cl], 1.42-2.65). Further, participants in the extremely lean subgroup within the underweight group had an even higher $\mathrm{OR}$ for depression $(\mathrm{OR}, 3.06 ; 95 \% \mathrm{Cl}$, 2.28-4.10). Regarding weight management methods, the rate of depression was higher for fasting and for skipping or reducing a meal than for exercise.

Conclusion: Biased BMI and body image perception are associated with increased incidence of depression. In addition, specific weight control methods, such as fasting, skipping a meal, and reducing food consumption, are also associated with increased incidence of depression.

Key words: Body mass index, Body image, Depression
Received October 13, 2018

Reviewed November 26, 2018

Accepted January 15, 2019

${ }^{*}$ Corresponding author

Soo Min Hong

https://orcid.org/0000-0003-0849-9785

Department of Internal Medicine, Seoul Paik Hospital, Inje University College of Medicine, 9 Mareunnae-ro, Jung-gu, Seoul 04551, Korea

Tel: +82-2-2270-0001

Fax: +82-2-2270-0579

E-mail: raisondetre@hanmail.net

\section{INTRODUCTION}

Obesity has been increasing worldwide over the last several years. In 2016, more than 650 million adults aged 18 years and older were obese, which is a $13 \%$ prevalence. ${ }^{1}$ The obesity rate is similarly increasing in Korea, where, in 2016, it was $34.8 \%$ (42.3\% in men, $26.4 \%$ in women) in adults aged 19 years and older. ${ }^{2}$ Obesity is a major health concern related to serious comorbidities, such as hypertension, dyslipidemia, and other cardiovascular diseases, as well as with mortality.-5 It also affects psychiatric health and economic burden. ${ }^{6,7}$ Underweight status also causes health and psychiatric problems. Low body mass index (BMI) is a well-known risk

Copyright (C) 2019 Korean Society for the Study of Obesity

(a) This is an Open Access article distributed under the terms of the Creative Commons Attribution Non-Commercial License (http://creativecommons.org/licenses/by-nc/4.0/) which permits unrestricted non-commercial use, distribution, and reproduction in any medium, provided the original work is properly cited. 
factor of osteoporosis ${ }^{8}$ and diabetes. ${ }^{9}$ Studies have shown that mortality is increased with being underweight. ${ }^{10,11}$

Furthermore, body image can influence BMI, behavior, self-esteem and interpersonal relationships. ${ }^{12}$ Body image is meaningful, in addition to actual BMI. ${ }^{13}$ Previously, we investigated the relationship between obesity and depression. ${ }^{14}$ In the present study, we investigate not only the association between $\mathrm{BMI}$ and depression, but also the relationships among body image perception, weight control method, and depression.

\section{METHODS}

\section{Study population}

In this study, we used a randomly selected population from the categorized nationwide data of the Sixth and Seventh Korea National Health and Nutrition Examination Survey (KNHANES VI2, VII-1) 2014 and 2016, conducted by the Korea Centers for Disease Control and Prevention (KCDC).

Data from 15,700 participants were examined: 7,550 in 2014 and 8,150 in 2016. These years' data include nine-item Patient Health Questionnaire (PHQ-9) scores measuring depression. After we excluded participants who were 18 years or younger in age, missing BMI data, or pregnant, 11,782 participants were included in the final analysis.

All participants provided informed consent before data collection, and the survey was approved by the KCDC Bioethics Committee (approval No. 2013-12EXP-03-5C in 2014) and the Institutional Review Board of Inje University Seoul Paik Hospital (IRB No. PAIK 2018-09-002).

\section{Clinical variables}

We classified the participants into four groups according to BMI: underweight $\left(\mathrm{BMI}<18.5 \mathrm{~kg} / \mathrm{m}^{2}\right)$, normal-to-overweight (BMI $18.5-24.9 \mathrm{~kg} / \mathrm{m}^{2}$ ), obesity stage I (BMI $25.0-29.9 \mathrm{~kg} / \mathrm{m}^{2}$ ), and obesity stage II $\left(\mathrm{BMI}>30.0 \mathrm{~kg} / \mathrm{m}^{2}\right)$, based on the Korean Society for the Study of Obesity practice guidelines. ${ }^{2,15}$

KNHANES of 2014 and 2016 included a self-PHQ-9 depression-screening test. PHQ-9 scores measure depression symptoms and are also used as a diagnostic criterion in the Diagnostic and Statistical Manual of Mental Disorders, fourth edition, for depres- sive disorders. ${ }^{16-18}$ We defined a PHQ-9 score of 10 or higher as indicating depression.

We also measured sociodemographic variables of age, sex, educational level, household income, residential area, and marital status. Level of education was classified as elementary or lower $(\leq 6$ years of school), middle school ( $\leq 9$ years of school), high school (1012 years of school), and college or above ( $\geq 13$ years of school). For household income, monthly household income was divided by the number of family members and classified into quantiles. Area of residence was divided into urban and rural. For marital status, those with a spouse or living with a partner were defined as married, and single participants were defined as never married.

The health behavior variables were smoking, drinking, periodic physical activity, and stress perception. Smoking status was categorized as current smoker, past smoker, and nonsmoker. Alcohol status was divided into two groups according to monthly drinking frequency in the past year: less than once a month and more than once a month. Regular physical activity was defined as performing more than 2.5 hours of moderate-intensity physical activity per week, more than 1.25 hours of high-intensity physical activity per week, or a corresponding amount of both moderate- and high-intensity physical activity ( 1 minute of high-intensity activity equaled 2 minutes of moderate-intensity activity) per week. Stress perception was classified as low or high.

Participants diagnosed with any type of cancer (stomach, liver, colon, breast, lung, uterine cervical, thyroid, and other) were classified into the cancer groups, and those who were diagnosed with any disease of hypertension, dyslipidemia, stroke, myocardial infarction, angina, diabetes, renal failure, liver cirrhosis, hepatitis B virus $(\mathrm{HBV})$, or hepatitis $\mathrm{C}$ virus $(\mathrm{HCV})$ were considered to have comorbidities.

\section{Statistical analysis}

All statistical analyses were performed using IBM SPSS version 22.0 (IBM Corp., Armonk, NY, USA). The study sample was stratified into four groups based on BMI, as described above. We used the chi-square test to analyze differences in variables from baseline characteristics. We expressed nominal variables as frequencies and percentages.

We applied multiple logistic regression analyses to estimate the 
odds ratios (ORs) and 95\% confidence intervals (CIs) between the BMI groups and depression, as well as between body image perception and depression. The included covariates were sex, age, education level, household income, habitation, marital status, comorbidities, smoking, alcohol drinking, physical activities, and stress perception, with and without BMI.

We performed a cross-sectional analysis to investigate the presence of depression (PHQ-9 $\geq 10$ ) according to subjective perceptions of body image. We performed Fisher exact test to investigate the presence of depression according to body-weight-management method across BMI groups. We considered the variables with $P$ values smaller than 0.05 to be statistically significant.

\section{RESULTS}

\section{Baseline characteristics}

The baseline characteristics of the study population according to BMI groups are shown in Table 1 . We found that $6.5 \%$ of the participants had depression with PHQ-9 scores of 10 or greater. In relation to BMI, the percentage of participants with depression was the highest in the underweight group (11.3\%) followed by the obesity stage II ( $8.3 \%)$, obesity stage I (6.3\%), and normal-tooverweight groups (6.2\%). The proportion of female participants was the highest in the underweight group (70.6\%). In general, the proportion of male participants increased with BMI. Regarding age, the percentage of participants in their 20s was the highest in the underweight group (27.7\%) followed by participants in their 30 s $(23.4 \%)$. The proportion of cancer prevalence was higher in the underweight and normal-to-overweight groups (2.5\% and $2.4 \%$, respectively) than in the obesity groups. The proportion of those with comorbidities (hypertension, dyslipidemia, stroke, myocardial infarction, angina, diabetes, renal failure, liver cirrhosis, $\mathrm{HBV}$, or HCV) was higher in the obesity groups (stage I, $41.4 \%$ and stage II, $42.0 \%$ ) than in the underweight group. Drinking frequency in the underweight and normal-to-overweight groups was low at less than once a month (66.5\% and 63.3\%, respectively) and high in the obesity groups at more than once a month (stage I, $46.6 \%$ and stage II, $50.9 \%$, respectively). The percentage of participants who did not engage in moderate or vigorous physical activity was high in all BMI groups. The proportion of stress perception was the highest in the underweight group (36.4\%).

\section{Relationship between BMI group and depression}

Table 2 shows the results of our logistic regression analyzing the risk for depression according to BMI. With reference to the normal-to-overweight group, the underweight group had the greatest risk for depression (OR, 1.94; 95\% CI, 1.42-2.65; $P<0.001)$. The obesity stage II group had the second greatest risk for depression (OR, 1.38; 95\% CI, 1.00-1.91; $P<0.05$ ), and although not statistically significant, the obesity stage I group had the third greatest risk for depression (OR, 1.02; 95\% CI, 0.85-1.22).

This finding that the underweight group had the greatest risk for depression is consistent in model 1 (adjusted for sex, age, educational level, household income, habitation, and marital status) and model 2 (adjusted for sex, age, educational level, household income, habitation, marital status, comorbidities, smoking, alcohol drinking, and stress perception; OR, 1.77; 95\% CI, 1.28-2.44; $P<0.001$ and OR, 2.38; 95\% CI, 1.45-3.88) (Table 2).

\section{Relationships among body image perception, weight control method, and depression}

We classified the participants having PHQ-9 scores of 10 or higher according to their BMI and surveyed their perceived body image. The underweight group tended to think that they were extremely lean (60\%) or slightly lean (30\%). Although most participants in the normal-to-overweight group perceived themselves to have normal body images (43.4\%), some in this group perceived themselves to be slightly obese (25.6\%), slightly lean (19.0\%), extremely obese (3.4\%), or extremely lean (8.5\%). The obesity stage I group mostly perceived themselves to be slightly obese (54.5\%) or extremely obese (28.3\%). The obesity stage II group tended to perceive themselves as being extremely obese (77.3\%) or slightly obese (18.2\%). As this show, participants' perceptions of their own body images tended to be consistent with actual BMI (kappa coefficient, 0.463) (Table 3).

Table 4 shows the results of our logistic regression analysis of depression risk according to perceived body image. With reference to a perception of normal body image, the risk of depression was the highest for perceptions of extremely lean, followed by extremely obese, slightly lean, and slightly obese (OR, 3.06; 95\% CI, 2.28- 
Table 1. Baseline characteristics according to body mass index groups $(n=11,782)$

\begin{tabular}{|c|c|c|c|c|c|c|}
\hline Variable & Underweight & Normal-to-overweight & Obesity stage I & Obesity stage II & Total & $P$ \\
\hline PHO 9 score & & & & & & $<0.001$ \\
\hline$<10$ & $392(88.7)$ & $6,279(93.8)$ & $2,849(93.7)$ & $484(91.7)$ & $10,004(93.5)$ & \\
\hline$\geq 10$ & $50(11.3)$ & $413(6.2)$ & $191(6.3)$ & $44(8.3)$ & $698(6.5)$ & \\
\hline Sex & & & & & & $<0.001$ \\
\hline Male & $143(29.4)$ & 2,933 (39.9) & $1,699(50.4)$ & $247(43.4)$ & $5,022(42.6)$ & \\
\hline Female & $344(70.6)$ & $4,421(60.1)$ & $1,673(49.6)$ & $322(56.6)$ & $6,760(57.4)$ & \\
\hline Age (yr) & & & & & & $<0.001$ \\
\hline $19-29$ & $135(27.7)$ & $889(12.1)$ & $241(7.1)$ & $70(12.3)$ & $1,335(11.3)$ & \\
\hline $30-39$ & $114(23.4)$ & $1,281(17.4)$ & $536(15.9)$ & $119(20.9)$ & $2,050(17.4)$ & \\
\hline $40-49$ & $71(14.6)$ & 1,338(18.2) & 599 (17.8) & $113(19.9)$ & $2,121(18.0)$ & \\
\hline $50-59$ & $47(9.7)$ & $1,365(18.6)$ & $679(20.1)$ & $98(17.2)$ & $2,189(18.6)$ & \\
\hline $60-69$ & $35(7.2)$ & $1,189(16.2)$ & $672(19.9)$ & $94(16.5)$ & $1,990(16.9)$ & \\
\hline$\geq 70$ & $85(17.5)$ & $1,292(17.6)$ & $645(19.1)$ & $75(13.2)$ & $2,097(17.8)$ & \\
\hline Education (school year) & & & & & & $<0.001$ \\
\hline Elementary $(\leq 6)$ & $72(16.1)$ & $1,417(21.0)$ & $847(27.6)$ & $134(25.4)$ & $2,470(22.9)$ & \\
\hline Junior high $(\leq 9)$ & $30(6.7)$ & $709(10.5)$ & $343(11.2)$ & $53(10.1)$ & $1,135(10.5)$ & \\
\hline Senior high (10-12) & $160(35.9)$ & 2,204 (32.7) & 940 (30.7) & $186(35.3)$ & $3,490(32.4)$ & \\
\hline College $(\geq 13)$ & $184(41.3)$ & 2,414 (35.8) & $936(30.5)$ & 154 (29.2) & $3,688(34.2)$ & \\
\hline Household income & & & & & & $<0.001$ \\
\hline Low & $146(30.2)$ & $1,752(24.0)$ & $833(24.8)$ & 184 (32.4) & $2,915(24.9)$ & \\
\hline Middle low & $100(20.7)$ & $1,841(25.2)$ & 881 (26.2) & $137(24.1)$ & $2,959(25.2)$ & \\
\hline Middle high & $136(28.1)$ & $1,812(24.8)$ & $876(26.1)$ & $122(21.5)$ & $2,946(25.1)$ & \\
\hline High & $102(21.1)$ & $1,910(26.1)$ & $770(22.9)$ & $125(22.0)$ & $2,907(24.8)$ & \\
\hline Residential area & & & & & & $<0.001$ \\
\hline Urban & $397(81.5)$ & $5,990(81.5)$ & $2,638(78.2)$ & 439 (77.2) & 9,464 (80.3) & \\
\hline Rural & 90 (18.5) & $1,364(18.5)$ & 734 (21.8) & $130(22.8)$ & $2,318(19.7)$ & \\
\hline Marital status & & & & & & $<0.001$ \\
\hline Married & $328(67.4)$ & 6,221 (84.6) & $3,003(89.1)$ & 467 (82.1) & $10,019(85.0)$ & \\
\hline Never married & $159(32.6)$ & $1,132(15.4)$ & $369(10.9)$ & $102(17.9)$ & $1,762(15.0)$ & \\
\hline Comorbidity & & & & & & $<0.001$ \\
\hline No & 431 (90.0) & $5,309(73.7)$ & 1,931 (58.6) & $323(58.0)$ & 7,994 (69.3) & \\
\hline Yes & $48(10.0)$ & $1,899(26.3)$ & $1,363(41.4)$ & 234 (42.0) & $3,544(30.7)$ & \\
\hline Cancer & & & & & & 0.04 \\
\hline No & $467(97.5)$ & $7,037(97.6)$ & $3,243(98.5)$ & $548(98.4)$ & $11,295(97.9)$ & \\
\hline Yes & $12(2.5)$ & $171(2.4)$ & $51(1.5)$ & $9(1.6)$ & $243(2.1)$ & \\
\hline Smoking & & & & & & $<0.001$ \\
\hline Non-smoker & $301(65.2)$ & 4,430 (63.3) & $1,776(55.7)$ & $322(58.5)$ & $6,829(61.0)$ & \\
\hline Ex-smoker & $76(16.5)$ & $1,313(18.8)$ & 751 (23.6) & $101(18.4)$ & $2,241(20.0)$ & \\
\hline Current smoker & $85(18.4)$ & $1,253(17.9)$ & $661(20.7)$ & $127(23.1)$ & $2,126(19.0)$ & \\
\hline Smoking amount & & & & & & $<0.001$ \\
\hline$<10$ Cigarettes/day & $31(36.5)$ & $314(25.1)$ & $118(17.9)$ & $34(26.8)$ & $497(23.4)$ & \\
\hline$\geq 10$ Cigarettes/day & $54(63.5)$ & $939(74.9)$ & 542 (82.1) & $93(73.2)$ & 1,628 (76.6) & \\
\hline Alcohol & & & & & & $<0.001$ \\
\hline$<1$ Drink/mo & $208(66.5)$ & $3,183(63.3)$ & $1,197(53.4)$ & $187(49.1)$ & $4,775(60.0)$ & \\
\hline$\geq 1$ Drink/mo & $105(33.5)$ & $1,842(36.7)$ & $1,043(46.6)$ & $194(50.9)$ & $3,184(40.0)$ & \\
\hline Periodic physical activity & & & & & & 0.002 \\
\hline No & $255(57.6)$ & $3,405(50.6)$ & $1,612(52.7)$ & $296(56.1)$ & $5,568(51.7)$ & \\
\hline Yes & $188(42.4)$ & $3,328(49.4)$ & $1,449(47.3)$ & $232(43.9)$ & $5,197(48.3)$ & \\
\hline Stress perception & & & & & & $<0.001$ \\
\hline Low & $293(63.6)$ & $5,245(75.0)$ & $2,379(74.7)$ & $392(71.3)$ & 8,309 (74.3) & \\
\hline High & $168(36.4)$ & $1,747(25.0)$ & $806(25.3)$ & $158(28.7)$ & $2,879(25.7)$ & \\
\hline
\end{tabular}

Values are presented as number (\%). $P$-values were obtained by chi-square test.

PHQ-9, nine-item Patient Health Questionnaire. 
Table 2. Logistic regression analysis for BMl group and depression

\begin{tabular}{|c|c|c|c|c|c|c|}
\hline Variable & Crude OR (95\% CI) & $P$ & Adjusted OR $(95 \% \mathrm{Cl})^{*}$ & $P$ & Adjusted OR $(95 \% \mathrm{CI})^{\dagger}$ & $P$ \\
\hline Normal-to-overweight & 1.00 & - & 1.00 & - & 1.00 & - \\
\hline Underweight & $1.94(1.42-2.65)$ & $<0.001$ & $1.77(1.28-2.44)$ & $<0.001$ & $2.38(1.45-3.88)$ & 0.07 \\
\hline Obesity stage I & $1.02(0.85-1.22)$ & 0.83 & $1.00(0.84-1.20)$ & 0.99 & $0.73(0.50-1.06)$ & 0.04 \\
\hline Obesity stage II & $1.38(1.00-1.91)$ & $<0.05$ & $1.25(0.90-1.74)$ & 0.18 & $1.21(0.62-2.33)$ & 0.41 \\
\hline
\end{tabular}

${ }^{*}$ Adjusted for sex, age, educational level, household income, habitation, and marital status; ${ }^{\dagger}$ Adjusted for sex, age, educational level, household income, habitation, marital status, comorbidities, smoking, alcohol drinking, and stress perception.

$\mathrm{BMI}$, body mass index; $\mathrm{OR}$, odds ratio; $\mathrm{Cl}$, confidence interval.

Table 3. Proportion of subjects with depression (PHO 9 scores $\geq 10$ ) according to body image perception and actual BMl

\begin{tabular}{lccccccc}
\hline Variable & Extremely lean & Slightly lean & Normal & Slightly obese & Extremely obese & Total & $P$ \\
\hline Underweight & $30(60.0)$ & $15(30.0)$ & $4(8)$ & 0 & $1(2.0)$ & $50(100)$ & $<0.001$ \\
Normal-to-overweight & $35(8.5)$ & $78(19.0)$ & $178(43.4)$ & $105(25.6)$ & $14(3.4)$ & $410(100)$ & $<0.001$ \\
Obesity stage I & $1(0.5)$ & 0 & $32(16.9)$ & $103(54.5)$ & $53(28.3)$ & $189(100)$ & $<0.001$ \\
Obesity stage II & 0 & 0 & $2(4.5)$ & $8(18.2)$ & $34(77.3)$ & $44(100)$ & 0.11 \\
\hline
\end{tabular}

Values are presented as number (\%). $P$-values were obtained by chi-square test.

$\mathrm{PHO}-9$, nine-item Patient Health Questionnaire; BMI, body mass index.

Table 4. Logistic regression analysis for body image perception and depression

\begin{tabular}{lcccccc}
\hline Variable & Crude OR $(95 \% \mathrm{CI})$ & $P$ & Adjusted OR $(95 \% \mathrm{Cl})^{*}$ & $P$ & ${\text { Adjusted OR }(95 \% \mathrm{CI})^{\dagger}}$ \\
\hline Normal & 1.00 & & 1.00 & & 1.00 & \\
Extremely lean & $3.06(2.28-4.10)$ & $<0.001$ & $2.37(1.69-3.31)$ & $<0.001$ & $1.27(0.56-2.91)$ & 0.57 \\
Slightly lean & $1.49(1.16-1.92)$ & $<0.05$ & $1.49(1.14-1.94)$ & $<0.05$ & $0.99(0.54-1.79)$ & 0.96 \\
Slightly obese & $1.16(0.96-1.41)$ & 0.13 & $1.30(1.04-1.61)$ & $<0.05$ & $0.96(0.56-1.65)$ & 0.88 \\
Extremely obese & $2.39(1.87-3.06)$ & $<0.001$ & $2.49(1.83-3.39)$ & $<0.001$ & $2.09(0.95-4.60)$
\end{tabular}

${ }^{*}$ Adjusted for sex, age, educational level, household income, habitation, marital status, and BMl; ${ }^{\dagger}$ Adjusted for sex, age, educational level, household income, habitation, marital status, comorbidities, smoking, alcohol drinking, stress perception, and BMI.

$\mathrm{OR}$, odds ratio; $\mathrm{Cl}$, confidence interval; $\mathrm{BMI}$, body mass index.

Table 5. Proportion of subjects with depression (PHO 9 scores $\geq 10$ ) according to weight control method and actual BMl

\begin{tabular}{lrrrrrr}
\hline Variable & Exercise & Fasting, skipping a meal, or reducing food consumption & Drug & One-food diet+etc. & Total & $P$ \\
\hline Underweight & $1(14.3)$ & $5(71.4)$ & $1(14.3)$ & 0 & $7(100)$ & 0.53 \\
Normal-to-overweight & $37(20.0)$ & $120(64.9)$ & $21(11.4)$ & $7(3.8)$ & $185(100)$ & $<0.05$ \\
Obesity stage I & $24(21.4)$ & $65(58.0)$ & $19(17.0)$ & $4(3.6)$ & $112(100)$ & 0.03 \\
Obesity stage II & $4(14.3)$ & $14(50.0)$ & $8(28.6)$ & $2(7.1)$ & $28(100)$ & 0.15 \\
\hline
\end{tabular}

Values are presented as number (\%). $P$-values were obtained by Fisher exact test.

$\mathrm{PHO}-9$, nine-item Patient Health Questionnaire; BMI, body mass index.

4.10 vs. OR, 2.39 ; $95 \%$ CI, $1.87-3.06$ vs. OR, 1.49 ; $95 \%$ CI, $1.16-$ 1.92 vs. OR, 1.16; 95\% CI, 0.96-1.41). In model 1 (adjusted for sex, age, educational level, household income, habitation, marital status, and BMI), the risk of depression was the highest for perceptions of extremely obese followed by extremely lean, slightly lean, and slightly obese (OR, 2.49; 95\% CI, 1.83-3.39 vs. OR, 2.37; 95\% CI, $1.69-3.31$ vs. OR, 1.49 ; 95\% CI, $1.14-1.94$ vs. OR, 1.30; $95 \%$
CI, 1.04-1.61; $P<0.05)$ (Table 4).

Regarding the presence of depression according to weight management method across BMI groups, the prevalence of depression was the highest for any manner of fasting, skipping a meal, or reducing the amount of food consumption. The prevalence of depression was next highest with exercise, followed by diet pills and one-food diets (Table 5). 


\section{DISCUSSION}

We had earlier examined the association between BMI and depression, finding that the risk for depression was higher in the underweight group than in the obesity groups. ${ }^{14}$ In the underweight group, the proportions of women and younger adults in their $20 \mathrm{~s}$ and 30s were high, as was the prevalence rate of cancer. Drinking frequency was low, and stress perception was the highest in the underweight group. These results seem to reflect the fact that younger participants in their 20s and 30s are affected by the social ideology preference for a thin — beyond slim-figure for women.

Our multivariate analyses revealed that the risk for depression was high in the underweight group and among participants who perceived themselves to be extremely lean or extremely obese. In addition to BMI, perception of body image significantly affected depression. In our analysis of the presence of depression according to weight control method, the prevalence of depression was the highest with fasting, skipping a meal, and reducing food consumption.

Among participants with depression, defined as a PHQ-9 score of 10 or higher, actual BMI and perceived body image were generally consistent. However, in contrast to our findings, some studies have found that actual BMI is not in accordance with perceived body image.

One Korean study investigated the degree of discordance between BMI and body image in adults using data from 172,132 participants in the 2010 Community Health Survey. About $25 \%$ of the participants showed a discordance between BMI and body image, $20 \%$ showed underestimation and 5\% showed overestimation. Furthermore, the discordant BMI and body image group reported low physical activity. ${ }^{19}$

In 2013, another study on perceived body image investigated 90 participants consisting of obese individuals planning to undergo obesity surgery at Soonchunhyang University Hospital in Seoul, obese individuals on a nonsurgical diet, and normal weight individuals. They found that body dissatisfaction was positively correlated with depression $(\mathrm{r}=0.17)$ and interpersonal relationship problems $(r=0.39)$ and negatively correlated with relationship self-efficacy $(\mathrm{r}=-0.14)$ and self-esteem $(\mathrm{r}=-0.41) .{ }^{12}$ In a 2011 study conducted at five elementary schools in Seoul, exercise frequency and prefer- ence dropped as participants perceived their body images to be more obese. Furthermore, those with body image dissatisfaction caused by discordance between actual and ideal body image had higher stress related to obesity, higher stress related to criticism among friends, higher fear of failure, lower exercise ability, and higher stress related to hot and vigorous exercise. ${ }^{20}$

A longitudinal study investigated body image and its impact on Chinese children, using the 2000-2011 China Health National Survey data. Compared to Chinese boys, a higher proportion of Chinese girls were in the lower actual BMI group, and they more often desired a thinner body. About $11 \%$ of the children attempted to lose weight and believed they engaged in little physical activity. ${ }^{21}$

Several previous studies reported that, compared to those who had a thin body image of themselves in childhood, people who perceived themselves as being fat in childhood showed more weight gain in addition to several other factors, such as exercise stress and low self-esteem, in adolescence..$^{22-26}$

In contrast, the above Chinese study found that having a fat body image helped children to realize the need for weight loss, attempt to go on a diet, and recognize that they engaged in little exercise, thereby serving as a source of self-motivation to achieve average weight. $^{21}$ The study suggests that appropriate body image perception motivates individuals to change their health behavior.

Currently, modern Korean society features a sociocultural trend to focus on appearance, and particularly, younger adults and women desire to have the ideal body figure featured in public media. Their dissatisfaction with their own body image increases when their actual body image is inconsistent with the socially ideal body image. Additionally, people try to monitor and control their bodies to adhere to the ideal standard shown in public media. This may cause mental stress and depression. Body image dissatisfaction is related to self-esteem and affects interpersonal relationships and social productivity.

Therefore, society needs to focus its attention and systematic support on attempts to achieve body weight that is healthy and not just esthetically pleasing. Cognitive behavioral therapy is one approach to help individuals to control weight healthily and continuously. $^{27}$

Our study is cross-sectional and has related limitations. Body figures as well as perception of body image may change over time, but 
we could not examine such changes because our study was not longitudinal. Two strengths of the study are our using data from a large, nationwide Korean-representative survey and that it is the first to study these variables in Korea.

Biased BMI and body image perception are associated with increased incidence of depression. In addition, specific weight control methods, such as fasting, skipping meals, and reducing food consumption, rather than exercise, are associated with increased incidence of depression. Therefore, healthy methods to establish healthy body image and maintain normal-range BMI should be considered.

\section{CONFLICTS OF INTEREST}

The authors declare no conflict of interest.

\section{REFERENCES}

1. World Health Organization. Obesity and overweight [Internet]. Geneva: World Health Organization; 2016 [cited 2018 Aug 29]. Available from: http://www.who.int/mediacentre/factsheets/fs311/en/

2. Korean Society for the Study of Obesity. Obesity Practice Guideline 2018. Seoul: Korean Society for the Study of Obesity; 2018.

3. Kim NS, Moon OR, Kang JH, Lee SY, Jeong BG, Lee SJ, et al. Increasing prevalence of obesity related disease for Koreans associated with overweight and obesity. Korean J Prev Med 2001;34:309-15.

4. The Health and Social Care Information Centre. Statistics on obesity, physical activity and diet. England: 2017 [Internet]. Leeds: NHS Digital; 2017 [cited 2018 Aug 30]. Available from: https://assets.publishing.service.gov.uk/government/uploads/ system/uploads/attachment_data/file/613532/obes-phys-acti-diet-eng-2017-rep.pdf

5. Guh DP, Zhang W, Bansback N, Amarsi Z, Birmingham CL, Anis AH. The incidence of co-morbidities related to obesity and overweight: a systematic review and meta-analysis. BMC Public Health 2009;9:88.

6. Ma J, Xiao L. Obesity and depression in US women: results from the 2005-2006 National Health and Nutritional Examination Survey. Obesity 2010;18:347-53.

7. Park JH, Yoon SJ, Lee HY, Cho HS, Lee JY, Eun SJ, et al. Estimating the burden of psychiatric disorder in Korea. J Prev Med Public Health 2006;39:39-45.

8. Kasper DL, Fauci AS, Hauser SL, Longo DL, Jameson JL, Loscalzo J. Harrison's principles of internal medicine. 19th ed. New York (NY): McGraw Hill; 2015.

9. Katanoda K, Noda M, Goto A, Mizunuma H, Lee JS, Hayashi $\mathrm{K}$. Being underweight in adolescence is independently associated with adult-onset diabetes among women: The Japan Nurses' Health Study.J Diabetes Investig 2018 Oct 5 [Epub]. https:// doi.org/10.1111/jdi.12947

10. Lee JY, Kim HC, Kim C, Park K, Ahn SV, Kang DR, et al. Underweight and mortality. Public Health Nutr 2016;19:1751-6.

11. Roh L, Braun J, Chiolero A, Bopp M, Rohrmann S, Faeh D, et al. Mortality risk associated with underweight: a census-linked cohort of 31,578 individuals with up to 32 years of follow-up. BMC Public Health 2014;14:371.

12. Kim HH, Baek JM, Hwang J, Kim YJ. A comparison of the psychological characteristics of obese people: the association between depression, body shape dissatisfaction and self-esteem of surgical treatment group and no treatment group. Korean J Obes 2014;23:32-40.

13. Schwartz MB, Brownell KD. Obesity and body image. Body Image 2004;1:43-56.

14. Hong SM, Hur YI. Relationship between obesity and depression in Korean adults: Korea National Health and Nutrition Examination Survey 2014. Medicine 2017;96:e9478.

15. WHO Expert Consultation. Appropriate body-mass index for Asian populations and its implications for policy and intervention strategies. Lancet 2004;363:157-63.

16. Han C, Jo SA, Kwak JH, Pae CU, Steffens D, Jo I, et al. Validation of the Patient Health Questionnaire-9 Korean version in the elderly population: the Ansan Geriatric study. Compr Psychiatry 2008;49:218-23.

17. American Psychiatric Association. Diagnostic and statistical manual of mental disorders. 4th ed. Washington (DC): American Psychiatric Association; 1994.

18. Kroenke K, Spitzer RL, Williams JB. The PHQ-9: validity of a 
brief depression severity measure. J Gen Intern Med 2001;16: 606-13.

19. Chun IA, Ryu SY, Park J, Han MA, Choi SW, Ko DS. The associations between discordance of body image and physical activities among adults aged 19 to 64 years: based on the data from 2010 community health survey. Korean J Obes 2014;23: 274-80.

20. Hong YS, Kim CS. The effect of body fat percentage, self body image, and stress of exercise on exercise participation in obese children. Korean J Obes 2012;21:203-12.

21. Min J, Yan AF, Wang VH, Wang Y. Obesity, body image, and its impact on children's eating and exercise behaviors in China: a nationwide longitudinal study. Prev Med 2018;106:101-6.

22. Robinson E, Sutin AR. Parental perception of weight status and weight gain across childhood. Pediatrics 2016;137:e20153957.

23. Liechty JM, Lee MJ. Body size estimation and other psychosocial risk factors for obesity onset among US adolescents: find- ings from a longitudinal population level study. Int J Obes 2015; 39:601-7.

24. Wang Y, Beydoun MA, Li J, Liu Y, Moreno LA. Do children and their parents eat a similar diet? Resemblance in child and parental dietary intake: systematic review and meta-analysis. J Epidemiol Community Health 2011;65:177-89.

25. Hunger JM, Tomiyama AJ. Weight labeling and obesity: a longitudinal study of girls aged 10 to 19 years. JAMA Pediatr 2014; 168:579-80.

26. Tomiyama AJ. Weight stigma is stressful: a review of evidence for the Cyclic Obesity/Weight-Based Stigma model. Appetite 2014;82:8-15.

27. Castelnuovo G, Pietrabissa G, Manzoni GM, Cattivelli R, Rossi A, Novelli M, et al. Cognitive behavioral therapy to aid weight loss in obese patients: current perspectives. Psychol Res Behav Manag 2017;10:165-73. 